\title{
Clouds in the atmospheres of extrasolar planets
}

\section{On the scattering greenhouse effect of $\mathrm{CO}_{2}$ ice particles: Numerical radiative transfer studies}

\author{
D. Kitzmann ${ }^{1}$, A. B. C. Patzer ${ }^{1}$, and H. Rauer ${ }^{1,2}$ \\ 1 Zentrum für Astronomie und Astrophysik, Technische Universität Berlin, Hardenbergstr. 36, 10623 Berlin, Germany \\ e-mail: kitzmann@astro.physik.tu-berlin.de \\ ${ }^{2}$ Institut für Planetenforschung, Deutsches Zentrum für Luft- und Raumfahrt (DLR), Rutherfordstr. 2, 12489 Berlin, Germany
}

Received 16 July 2012 / Accepted 17 June 2013

\begin{abstract}
Context. Owing to their wavelength-dependent absorption and scattering properties, clouds have a strong impact on the climate of planetary atmospheres. The potential greenhouse effect of $\mathrm{CO}_{2}$ ice clouds in the atmospheres of terrestrial extrasolar planets is of particular interest because it might influence the position and thus the extension of the outer boundary of the classic habitable zone around main sequence stars. Such a greenhouse effect, however, is a complicated function of the $\mathrm{CO}_{2}$ ice particles' optical properties. Aims. We study the radiative effects of $\mathrm{CO}_{2}$ ice particles obtained by different numerical treatments to solve the radiative transfer equation. To determine the effectiveness of the scattering greenhouse effect caused by $\mathrm{CO}_{2}$ ice clouds, the radiative transfer calculations are performed over the relevant wide range of particle sizes and optical depths, employing different numerical methods.

Methods. We used Mie theory to calculate the optical properties of particle polydispersion. The radiative transfer calculations were done with a high-order discrete ordinate method (DISORT). Two-stream radiative transfer methods were used for comparison with previous studies.

Results. The comparison between the results of a high-order discrete ordinate method and simpler two-stream approaches reveals large deviations in terms of a potential scattering efficiency of the greenhouse effect. The two-stream methods overestimate the transmitted and reflected radiation, thereby yielding a higher scattering greenhouse effect. For the particular case of a cool M-type dwarf, the $\mathrm{CO}_{2}$ ice particles show no strong effective scattering greenhouse effect by using the high-order discrete ordinate method, whereas a positive net greenhouse effect was found for the two-stream radiative transfer schemes. As a result, previous studies of the effects of $\mathrm{CO}_{2}$ ice clouds using two-stream approximations overrated the atmospheric warming caused by the scattering greenhouse effect. Consequently, the scattering greenhouse effect of $\mathrm{CO}_{2}$ ice particles seems to be less effective than previously estimated. In general, higher order radiative transfer methods are needed to describe the effects of $\mathrm{CO}_{2}$ ice clouds accurately as indicated by our numerical radiative transfer studies.
\end{abstract}

Key words. planets and satellites: atmospheres - scattering - radiative transfer

\section{Introduction}

Clouds can have a strong impact on the climate of terrestrial planetary atmospheres by either scattering the incident stellar radiation back to space (albedo effect) or by trapping the infrared radiation in the atmosphere (greenhouse effect). The extension of the habitable zone around different types of stars depends, therefore, on the presence of clouds (see e.g. Kasting et al. 1993; Selsis et al. 2007). The position of the inner boundary of the habitable zone is determined by the efficiency of the albedo effect by water droplet clouds (Kasting 1988). The outer boundary, on the other hand, might be influenced by the formation of $\mathrm{CO}_{2}$ ice clouds and their corresponding climatic impact (e.g. Kasting et al. 1993; Forget \& Wordsworth 2010). Selsis et al. (2007) discuss the potential effects of $\mathrm{CO}_{2}$ clouds for the outer boundary of the habitable zone around the star Gliese 581. In particular the planet Gliese 581d has been studied with one and three-dimensional atmospheric models by Wordsworth et al. (2010b, 2011), which included a simplified model for describing $\mathrm{CO}_{2}$ clouds. Their results show that the $\mathrm{CO}_{2}$ clouds contribute to the greenhouse effect by increasing the surface temperature of Gliese 581d.
The effects of $\mathrm{CO}_{2}$ ice clouds, however, differ from those of water droplets or ice clouds due to the very different optical properties. Additionally, whether the greenhouse or albedo effect dominates for a given cloud of $\mathrm{CO}_{2}$ ice crystals depends on a variety of cloud properties. Apart from the wavelength-dependent optical depths, the crystal size is the most important quantity for determining the climatic effect of $\mathrm{CO}_{2}$ clouds (Pierrehumbert \& Erlick 1998; Forget \& Pierrehumbert 1997).

The only $\mathrm{CO}_{2}$ clouds observed so far in planetary atmospheres are found in the atmosphere of Mars. Most of the observed clouds near the Martian equator are composed of particles with rather small effective radii $\left(a_{\mathrm{eff}}<3 \mu \mathrm{m}\right)$ and with optical depths below 0.5 in the visible wavelength region (Määttänen et al. 2010; Montmessin et al. 2007; Vincendon et al. 2011). Larger particles $\left(a_{\mathrm{eff}} \sim 10 \mu \mathrm{m}\right)$ are found at high latitudes during the polar night (Forget et al. 1995; Colaprete et al. 2003), for example.

Clouds composed of $\mathrm{CO}_{2}$ ice crystals are also thought to play a major role in the conditions of the early Martian atmosphere. For a dense early Mars atmosphere, Forget \& Pierrehumbert (1997) have shown that $\mathrm{CO}_{2}$ ice clouds can exhibit a greenhouse effect by scattering of infrared radiation if the effective 


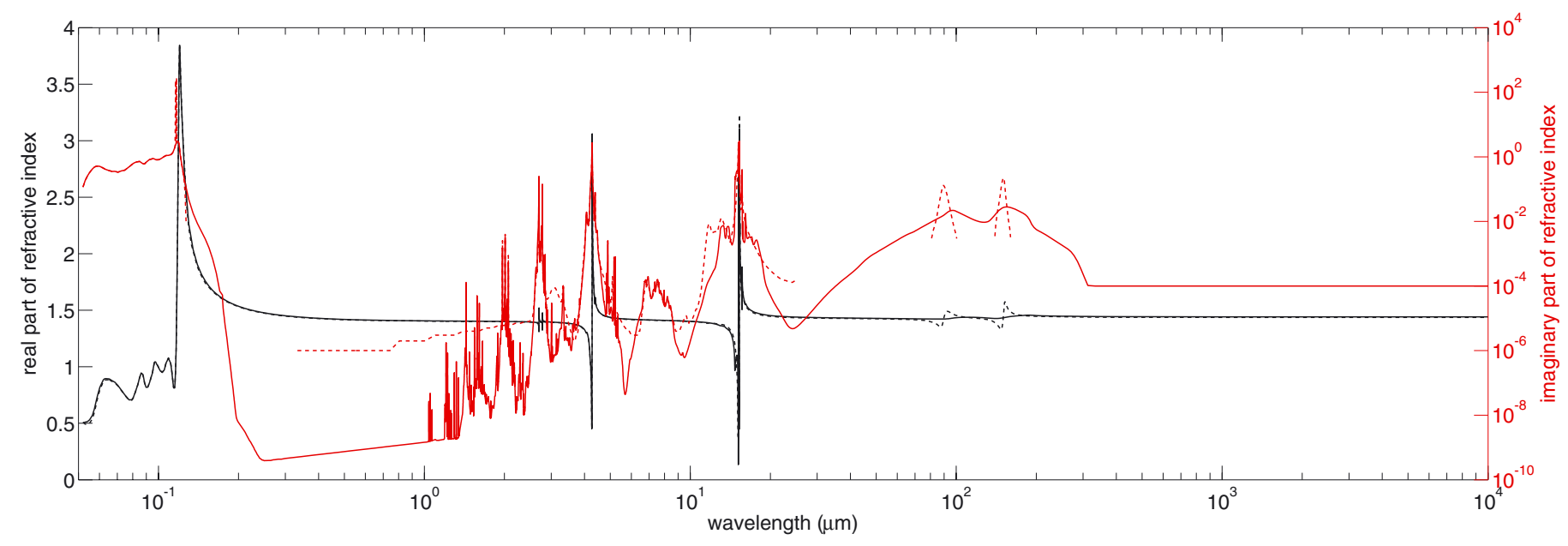

Fig. 1. Real part (black line) and imaginary part (red line) of the refractive index of $\mathrm{CO}_{2}$ ice. The solid lines indicate the data published by Hansen $(1997,2005)$, the dashed lines mark the data compilation from Warren (1986).

particle radii are larger than $\sim 6 \mu \mathrm{m}$. Smaller particle radii yield a dominating albedo effect that leads to lower surface temperatures. A similar approach for early Mars, but including a more detailed radiative transfer treatment, was used by Mischna et al. (2000). Their results indicate that the altitude of $\mathrm{CO}_{2}$ clouds influence the efficiency of the greenhouse effect. The formation of $\mathrm{CO}_{2}$ clouds in the atmosphere of early Mars has been studied by Colaprete \& Toon (2003) with a detailed microphysical model using laboratory data from Glandorf et al. (2002) for the description of the $\mathrm{CO}_{2}$ ice nucleation. The microphysical model was coupled with a time-dependent one-dimensional radiativeconvective atmospheric model. According to their model calculations, the mean particle size in a dense $\mathrm{CO}_{2}$-dominated atmosphere of early Mars is of the order of $1000 \mu \mathrm{m}$. The results of Colaprete \& Toon (2003) also indicate that the (scattering) greenhouse effect is limited overall by the evaporation of $\mathrm{CO}_{2}$ ice particles due to the large amount of latent heat released during their formation. The greenhouse effect of such $\mathrm{CO}_{2}$ clouds is thereby self-limited. Besides early Mars, $\mathrm{CO}_{2}$ clouds may have also contributed to the climate of the early Earth (see e.g. Caldeira \& Kasting 1992).

In this paper we study the radiative effects of $\mathrm{CO}_{2}$ ice particles. Mie theory is used to calculate the optical properties for assumed gamma particle size distributions for a broad range of effective radii (Sect. 2). In Sect. 3 discrete ordinate radiative transfer schemes are applied to obtain the spectral reflectance and transmittance of given $\mathrm{CO}_{2}$ clouds for different optical depths. The net radiative effects of such $\mathrm{CO}_{2}$ ice clouds for the particular case of a cool M-type dwarf star are discussed in Sect. 4. To make a comparison with previous model studies on the climatic effects of $\mathrm{CO}_{2}$ ice clouds, we also apply twostream radiative transfer methods. These additional calculations are compared with our findings obtained from a high-order discrete ordinate method.

\section{Optical properties of $\mathrm{CO}_{2}$ ice particles}

\subsection{Refractive index of $\mathrm{CO}_{2}$ ice}

Two different (main) compilations of the refractive index are available for $\mathrm{CO}_{2}$ ice covering the wavelength range from the far UV up to the microwave region and are shown in Fig. 1. The first set of this refractive index was published by Warren (1986). The imaginary part was compiled from various published measurements by different authors, connected by extrapolation and interpolation. Gaps in the data are present in the visible and far-infrared (FIR) wavelength range (see Fig. 1) where no published measurements were available at that time. Based on the compiled imaginary part, Warren (1986) calculated the corresponding real part by using the Kramers-Kronig relations (Kramers 1927; Kronig 1926).

The second compilation was published by Hansen (1997, $2005)$. For the imaginary part of the refractive index laboratory measurements by Hansen were used from $0.174 \mu \mathrm{m}$ up to $333 \mu \mathrm{m}$. Below $0.174 \mu \mathrm{m}$ and above $333 \mu \mathrm{m}$, the compilation was extended with data from Warren (1986). The corresponding real part of the refractive index was also obtained via the Kramers-Kronig relations.

The imaginary part of the refractive index is directly related to the absorption coefficient. For $\mathrm{CO}_{2}$ it shows considerable variations of about twelve orders of magnitudes throughout the whole wavelength range. Especially in the IR it exhibits a complicated oscillatory behaviour with several strong absorption bands. Besides these strong bands, the imaginary part suggests that the overall absorption of $\mathrm{CO}_{2}$ ice seems to be small in the IR in comparison to, say, water ice (see Warren \& Brandt 2008, for details on the refractive index of $\mathrm{H}_{2} \mathrm{O}$ ice). Compared to the imaginary part, the real part does not show any such large variations. It, however, has two distinct large features in the IR near $4.3 \mu \mathrm{m}$ and $15.2 \mu \mathrm{m}$.

Besides these two large data compilations, other measurements are also available for the astrophysically important strong absorption bands in the IR and FIR. The refractive index for these bands was published by Johnson \& Atreya (1996), Hudgins et al. (1993), Ehrenfreund et al. (1996), or Baratta \& Palumbo (1998), among others. The wavelengths positions of these absorption bands agree overall with those of the Hansen (1997, 2005) and Warren (1986) data compilations. However, the band widths and heights are strong functions of temperature and can, therefore, vary between the different measurements. For this study the refractive index from Hansen $(1997,2005)$ is used.

\subsection{Mie theory calculations}

To calculate the optical properties of $\mathrm{CO}_{2}$ ice particles Mie theory (Mie 1908) is used in this study by assuming a spherical particle shape. From observations in the Earth atmosphere, it 
is well known that water-ice crystals are rarely spherical but show a broad distribution of different particle shapes. In contrast to $\mathrm{H}_{2} \mathrm{O}$, the unit cell of $\mathrm{CO}_{2}$ ice has a face-centred cubic structure. Therefore, as found in in-situ laboratory measurements by Behnken (1912), Wahl (1913), or Wergin et al. (1997), for instance, $\mathrm{CO}_{2}$ crystals can have cubic or octahedral shapes. Combinations of both (cuboctahedra) or more complicated shapes also occur, such as rhobic-dodecahedral crystals. In-situ measurements of the shapes of $\mathrm{CO}_{2}$-ice cloud particles in, for example, the Martian atmosphere are not yet available. Furthermore, the scattering properties of such non-spherical particles are extremely demanding to calculate so that we use the approximation of spherical particles in this study, which has also been done in all previous studies on the climatic impact of $\mathrm{CO}_{2}$ ice clouds.

Following the approach of Forget \& Pierrehumbert (1997), we describe the size distribution of the cloud particles by a modified gamma distribution:

$f(a)=\frac{\left(a_{\mathrm{eff}} v\right)^{2-1 / v}}{\Gamma\left(\frac{1-2 v}{v}\right)} a^{\left(\frac{1}{v}-3\right)} \mathrm{e}^{-\frac{a}{a_{\mathrm{eff}}}}$

where $a_{\text {eff }}$ is the effective radius, $v$ the effective variance, and $\Gamma$ the gamma function. For the effective variance we adopt the value $v=0.1$ also used by Forget \& Pierrehumbert (1997). The effective radii are varied between $0.1 \mu \mathrm{m}$ and $200 \mu \mathrm{m}$ in this study. Mie theory (Wiscombe 1980, 1979) is used to obtain the optical properties for a distinct single particle size. These optical properties are then averaged over the assumed size distribution functions. The optical properties from Mie scattering calculations are shown in Fig. 2 for four different exemplary effective particle radii and an optical depth ${ }^{1}$ of $\tau=1$.

The optical depth of the larger particles is almost constant from the EUV up to the EHF wavelength region. This corresponds directly to the large-particle limit of Mie theory (Bohren \& Huffman 1998), which predicts a constant value for the extinction efficiency independent of the refractive index and, therefore, from the considered material. For the largest particles $\left(a_{\mathrm{eff}}=\right.$ $200 \mu \mathrm{m}$ ), this limit is reached for almost the whole wavelength range up to the FIR (Fig. 2). For each size distribution the optical depths show a distinct maximum at different wavelengths. This maximum value is located near the wavelengths roughly corresponding to the particle size. For example, the particles with $a_{\text {eff }}=20 \mu \mathrm{m}\left(a_{\mathrm{eff}}=1 \mu \mathrm{m}\right)$ have their maximum optical depth in the IR at $\lambda=27 \mu \mathrm{m}(\lambda=1.2 \mu \mathrm{m})$. The positions of these maxima will largely determine the radiative effects of the $\mathrm{CO}_{2}$ ice particles.

The single scattering albedo is shown in the middle panel of Fig. 2. The resulting albedo values indicate that scattering dominates over a wide wavelength range for the large $\mathrm{CO}_{2}$-ice crystals. Independent of the particle size, the single scattering albedo is almost unity from the near UV up to the NIR. As a direct consequence the incident stellar radiation will mostly be scattered and not absorbed at this wavelength range.

The albedo of $\mathrm{CO}_{2}$ ice in the IR shows complicated behaviour as a function of the effective particle size. Within the strong absorption bands, the albedo can be as low as zero, indicating dominating absorption. However, outside of these bands the scattering albedo of the $200 \mu \mathrm{m}$ and $20 \mu \mathrm{m}$ particle size distributions are almost unity up to the EHF and FIR, respectively. The particles with $a_{\mathrm{eff}}=0.1 \mu \mathrm{m}$ show much stronger variations in the single scattering albedo than the distributions with

\footnotetext{
1 Unless otherwise stated $\tau$ refers to the particular wavelength of $\lambda=$ $0.1 \mu \mathrm{m}$.
}

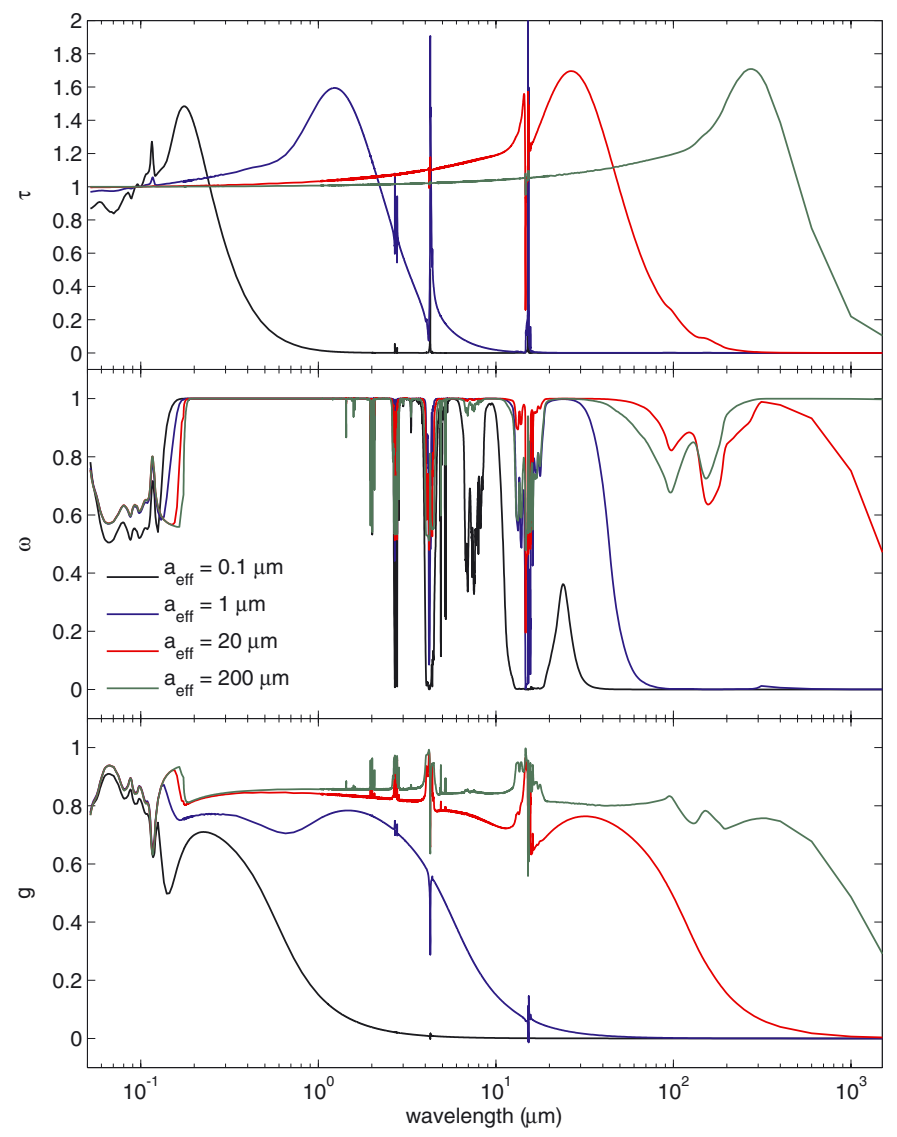

Fig. 2. Calculated optical properties of $\mathrm{CO}_{2}$ for four different size distributions: $a_{\mathrm{eff}}=0.1 \mu \mathrm{m}$ (black line), $a_{\mathrm{eff}}=1 \mu \mathrm{m}$ (blue line), $a_{\mathrm{eff}}=$ $20.0 \mu \mathrm{m}$ (red line), $a_{\mathrm{eff}}=200 \mu \mathrm{m}$ (green line). Upper diagram: optical depth, middle diagram: single scattering albedo $\omega$, lower diagram: asymmetry parameter $g$.

larger effective radii. In the IR, however, the single scattering albedo quickly approaches a value of almost zero for these small particles.

The single scattering albedo emphasises that the interaction of the radiation field with $\mathrm{CO}_{2}$-ice particles is quite different compared to, say, water-ice clouds. While water-ice clouds exhibit a greenhouse effect by absorption and re-emission of thermal radiation, $\mathrm{CO}_{2}$-ice particles will affect the thermal radiation mostly by scattering processes. To produce a corresponding (scattering) greenhouse effect (Forget \& Pierrehumbert 1997) the thermal radiation must be scattered back to the planetary surface by the $\mathrm{CO}_{2}$-ice clouds. Thus, the efficiency of the greenhouse effect of $\mathrm{CO}_{2}$ ice also depends on the mean scattering angle and, therefore, on the value of the asymmetry parameter.

The asymmetry parameters averaged over the size distributions are shown in the lower panel of Fig. 2. The resulting asymmetry parameters are always larger than or equal to zero (Rayleigh scattering limit), which indicates dominating forward scattering. For the largest particles with $a_{\mathrm{eff}}=200 \mu \mathrm{m}$ the asymmetry parameter has almost a constant value of about 0.9 from the UV up to the FIR except for strong variations in the absorption bands. With a value of about 0.8 , the asymmetry for $a_{\mathrm{eff}}=20 \mu \mathrm{m}$ is smaller in the wavelength regime from the UV up to the NIR. It then steadily increases up to 0.9 in the MIR before slowly declining for wavelength $\lambda>30 \mu \mathrm{m}$. Only for wavelengths larger than $300 \mu \mathrm{m}$ the asymmetry parameter approaches the limit of Rayleigh scattering $(g=0)$ as expected. The smallest particles considered here $\left(a_{\mathrm{eff}}=0.1 \mu \mathrm{m}\right)$ show a constant decline 
in $g$ already at $\lambda=0.3 \mu \mathrm{m}$ and approach the Rayleigh limit in the NIR.

\section{Radiative transfer calculations}

Radiative transfer calculations are performed for each particle size distribution to determine the radiative effects of the considered $\mathrm{CO}_{2}$-ice particles. In these calculations only one single cloud layer is studied, assuming plane-parallel geometry.

We consider the radiative transfer equation:

$\mu \frac{\mathrm{d} I_{\lambda}}{\mathrm{d} \tau_{\lambda}}=I_{\lambda}-S_{\lambda, *}\left(\tau_{\lambda}\right)-\omega_{\lambda} \frac{1}{2} \int_{-1}^{+1} p_{\lambda}\left(\mu, \mu^{\prime}\right) I_{\lambda}\left(\mu^{\prime}\right) \mathrm{d} \mu^{\prime}$

with the scattering phase function $p_{\lambda}$, the single scattering albedo $\omega_{\lambda}$, and the contribution due to an external illumination by a central star $S_{\lambda, *}\left(\tau_{\lambda}\right)$. The scattering phase function can be represented as an infinite series of Legendre polynomials (Chandrasekhar 1960)

$p_{\lambda}\left(\mu, \mu^{\prime}\right)=\sum_{n=0}^{\infty}(2 n+1) P_{n}(\mu) P_{n}\left(\mu^{\prime}\right) \chi_{\lambda, n}$

with the Legendre polynomials $P_{n}(\mu)$ and the phase function moments $\chi_{\lambda, n}$. In practice the series is truncated at a certain $n=N_{\max }$. For discrete ordinate methods the number of moments $N_{\text {max }}$ used to describe the phase function expansion series is a direct function of the number of ordinates (streams) considered (Chandrasekhar 1960).

In this study the scattering phase function is approximated by the Henyey-Greenstein function (Henyey \& Greenstein 1941). This phase function only depends on the asymmetry parameter $g$ and is an approximation of the full Mie scattering phase function. Although lacking many detailed features and complicated structure of the Mie phase function, the Henyey-Greenstein function preserves its average quantities, most notably its asymmetry parameter. The Henyey-Greenstein phase function is usually a good replacement for the much more complicated Mie phase function in case of higher optical depths because the finer details of the Mie phase functions are effaced by the multiple scattering effects. Only the averaged quantities (such as the asymmetry parameter) then determine the overall properties of the radiation field, especially the angular integrated quantities of the intensity, such as the radiation flux. However, at very small optical depths, the results obtained by using the HenyeyGreenstein function may not be so accurate.

For the solution of the radiative transfer equation Eq. (2) we employ different discrete ordinate methods (Chandrasekhar 1960). In particular, we use the well established and very flexible atmospheric radiative transfer code DISORT (Stamnes et al. 1988). Following the previous studies of Mischna et al. (2000) or Colaprete \& Toon (2003), we additionally also use twostream methods for comparison, namely a $\delta$-Eddington quadrature method for the incident stellar radiation and a hemispheric two-stream method in the IR (see Toon et al. 1989, for details on these methods).

The simple two-stream solution methods use only one stream for the upward and one for the downward direction. In this case only the first two moments of the phase function expansion would enter into the radiative transfer calculations. However, even the simplest phase functions, such as the Rayleigh scattering phase function, require the first three moments of the expansion for an accurate representation. Therefore, the twostream methods, such as the $\delta$-Eddington quadrature method or the hemispheric mean two-stream radiative transfer, cannot use the usual expansion series but must rely on different, simplified approximations of the phase function. For example, the hemispheric mean method uses a phase function of $1+g$ in the forward and $1-g$ in the backward direction (Toon et al. 1989).

For applying of the more general discrete ordinate solver DISORT, we use here 24 streams in all calculations, i.e. $N_{\max }+$ $1=24$. This allows for a much better description of the phase function than in the case of the two-stream methods. Such an approach usually should give more accurate results for systems dominated by angular dependent radiative transfer processes such as in particular anisotropic scattering.

To make an estimate, the scattering and absorption behaviour of atmospheric $\mathrm{CO}_{2}$ molecules and of the planetary surface has been approximately included in the radiative transfer calculations for the stellar radiation with a wavelength-dependent albedo $a_{\mathrm{p}, \lambda}$ below the cloud layer. The albedo $a_{\mathrm{p}, \lambda}$ roughly describes the impact of an Earth-like surface with a 2 bar $\mathrm{CO}_{2}$ atmosphere as an example. Therefore, we assume a constant (measured) Earth-like surface albedo of 0.13 (Kitzmann et al. 2010), lowered to 0.1 to account for the NIR absorption of $\mathrm{CO}_{2}$ molecules. In addition to this constant albedo describing the impact of the planetary surface, we added a Rayleigh-scatteringlike albedo with the well-known $\lambda^{-4}$ dependence to account for molecular Rayleigh scattering. The Rayleigh scattering contribution is important for central stars with higher effective temperatures, whereas the influence of the (reduced) surface albedo is important for cooler stars. Figure 4 shows the resulting (clearsky) planetary albedo $a_{\mathrm{p}}$ as a function of the effective temperatures of the central stars. We note that the surface albedo and the amount of gas that influences the Bond albedo due to Rayleigh scattering can have a strong impact on the net effect of a $\mathrm{CO}_{2}$ cloud. For example, low surface albedos (e.g. due to oceans) will yield a smaller net greenhouse effect, while a more reflective, Martian-like surface albedo can result in an increase in the scattering greenhouse effect. As mentioned by Forget \& Pierrehumbert (1997), the thermal emission by the cloud itself would only make a minor contribution and is therefore neglected in our study.

A zenith angle for the incident stellar radiation of 60 degrees is used here, which corresponds to the mean zenith angle in a one-dimensional global average atmospheric model. As a result of our radiative transfer calculations we obtain the fraction of the incident stellar radiation in downward direction $\epsilon_{\mathrm{s}, \lambda}$ at the cloud base for each wavelength $\lambda$ by

$\epsilon_{\mathrm{s}, \lambda}=\frac{F_{\mathrm{s}, \mathrm{cb}, \lambda}^{\downarrow}}{F_{\mathrm{s}, \lambda}^{\downarrow}}$

where $F_{\mathrm{s}, \lambda}^{\downarrow}$ denotes the incident stellar radiation at the top of the atmosphere and $F_{\mathrm{s}, \mathrm{cb}, \lambda}^{\downarrow}$ the downward shortwave radiation flux at the cloud base. The flux $F_{\mathrm{s}, \mathrm{cb}, \lambda}^{\downarrow}$ is composed of the incident stellar light that is transmitted and forward scattered through the cloud layer, as well as contributions from multiple scattering of that shortwave radiation between the cloud base and the lower atmosphere/planetary surface (approximated here by the wavelength-dependent albedo $a_{\mathrm{p}, \lambda}$ ). Additionally, we obtain the spectral transmittance of the cloud layer and the percentage of thermal IR radiation scattered back towards the surface (spectral reflectance $\epsilon_{\mathrm{a}, \mathrm{r}, \lambda}$ of the cloud) given by

$\epsilon_{\mathrm{a}, \mathrm{r}, \lambda}=\frac{F_{\mathrm{a}, \mathrm{cb}, \lambda}^{\downarrow}}{F_{\mathrm{a}, \lambda}^{\uparrow}}$ 

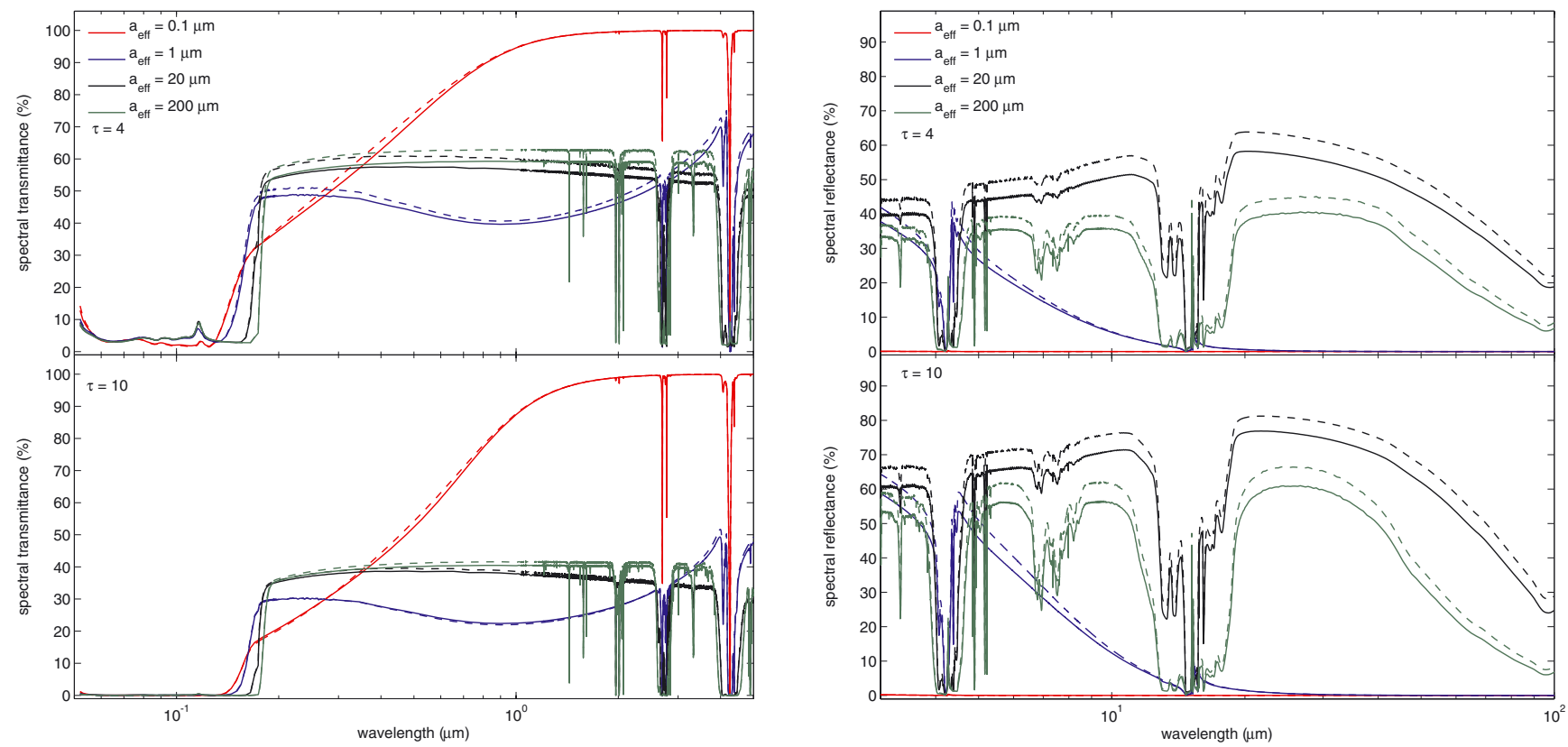

Fig. 3. Spectral transmittance (left column) and reflectance (right column) for two different optical depths: $\tau=4$ (upper panels) and $\tau=10$ (lower panels). Results are shown for four different size distributions: $a_{\mathrm{eff}}=0.1 \mu \mathrm{m}$ (red line), $a_{\mathrm{eff}}=1 \mu \mathrm{m}$ (blue line), $a_{\mathrm{eff}}=20.0 \mu \mathrm{m}$ (black line), and $a_{\mathrm{eff}}=200 \mu \mathrm{m}$ (green line). Calculations using DISORT (two-stream methods) are denoted by solid lines (dashed lines).

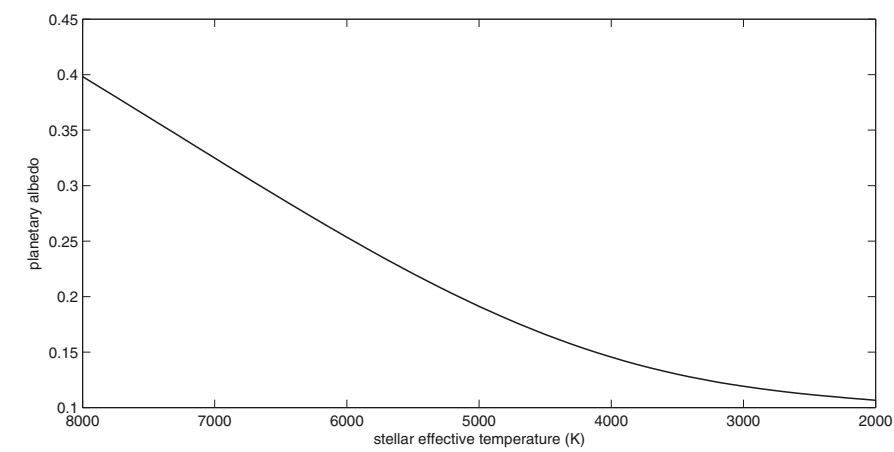

Fig. 4. Planetary albedo (clear-sky condition) as a function of the effective temperatures of the central stars.

where $F_{\mathrm{a}, \lambda}^{\uparrow}$ is the thermal radiation flux emitted from the atmosphere/surface and $F_{\text {a,cb }, \lambda}^{\downarrow}$ denotes the downward longwave flux at the cloud base. The incident fluxes for each wavelength are set to unity to simplify the numerical boundary conditions in the radiative transfer calculations. Obviously, this does not affect the computed values of the ratios $\epsilon_{\mathrm{a}, \mathrm{r}, \lambda}$ and $\epsilon_{\mathrm{s}, \lambda}$.

To illustrate the basic differences between the two-stream methods and DISORT, Fig. 3 summarises the resulting spectral transmittance and reflectance of the $\mathrm{CO}_{2}$ cloud for several different cloud properties. Additionally, the ratio $\epsilon_{\mathrm{s}, \lambda}$ is depicted in Fig. 5.

The smallest particles ( $\left.a_{\mathrm{eff}}=0.1 \mu \mathrm{m}\right)$ yield an almost $100 \%$ transmittance for wavelengths larger than $1 \mu \mathrm{m}$ even for a high optical depth of 10 . This implies that their effect on the incident stellar radiation will be small if the maximum of the stellar radiation is located in this wavelength region. On the other hand, they also have a reflectance of almost zero, which means that these small particles will not yield a scattering greenhouse effect (see Fig. 3).

The transmittance of larger particles is always less than $100 \%$ and decreases at higher optical depth. The strongest

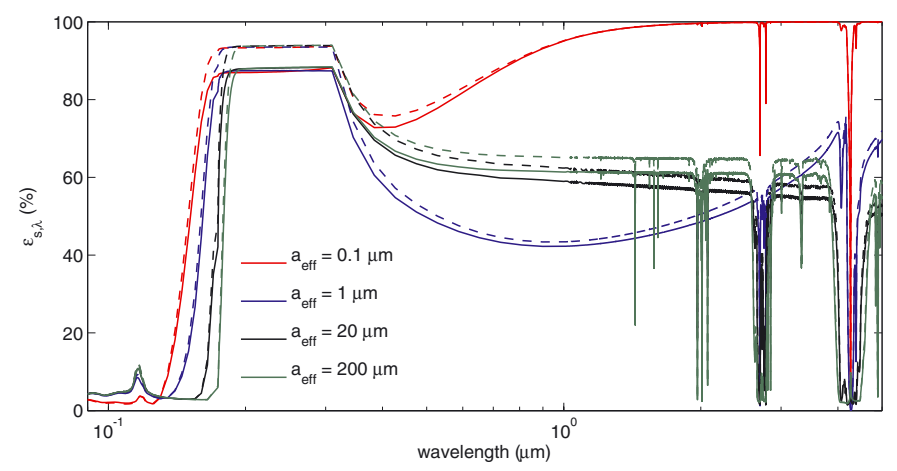

Fig. 5. Calculated fraction of the incident stellar radiation in downward direction at the cloud base $\left(\epsilon_{\mathrm{s}, \lambda}\right)$ for $\tau=4$. Results are shown for four different size distributions: $a_{\mathrm{eff}}=0.1 \mu \mathrm{m}$ (red line), $a_{\mathrm{eff}}=1 \mu \mathrm{m}$ (blue line), $a_{\mathrm{eff}}=20.0 \mu \mathrm{m}$ (black line), and $a_{\mathrm{eff}}=200 \mu \mathrm{m}$ (green line). Calculations using DISORT (two-stream methods) are denoted by solid lines (dashed lines).

negative impact on the transmittance is obtained for particles with $a_{\mathrm{eff}}=1 \mu \mathrm{m}$ because these particles have their largest contribution to the opacity in this wavelength region (see Fig. 2). The transmittance is almost equal for the largest particles. This is the result of the large-particle limit discussed in Sect. 2.2 yielding the same optical properties (optical depth, asymmetry parameter, and single scattering albedo) for all big particles at short wavelengths.

The spectral reflectance for the $a_{\mathrm{eff}}=1 \mu \mathrm{m}$ particles is very low (Fig. 3), especially in the wavelength region where most of the atmospheric thermal radiation is transported $(\lambda>8 \mu \mathrm{m})$. This and their strong negative impact on the transmittance suggests that these particles will more likely cool the lower atmosphere than cause a net scattering greenhouse effect.

However, larger particles can show high reflectance values in the IR wavelength range. The reflectance for $a_{\mathrm{eff}}=20 \mu \mathrm{m}$ is overall almost $20 \%$ higher than for the largest particles with $a_{\mathrm{eff}}=200 \mu \mathrm{m}$. This is caused by the higher optical depth of the 
$20 \mu \mathrm{m}$ particles in the thermal IR wavelength range (cf. Fig. 2) and, on the other hand, by the higher asymmetry parameter for $a_{\text {eff }}=200 \mu \mathrm{m}$, which leads to a larger fraction of the thermal radiation being scattered in forward direction to space rather than backwards to the planetary surface. This indicates that the scattering greenhouse effect will be most efficient for particles with sizes comparable to the wavelength of the thermal radiation.

Figures 3 and 5 also show the results of the two-stream methods. The results indicate that the two-stream methods overestimate both

- the transmission/scattering of stellar radiation, and

- and the back-scattering of thermal radiation.

For the $\delta$-Eddington two-stream method the differences are about $6 \%$ while the hemispheric mean two-stream yields deviations up to $20 \%$. Thus, two-stream methods will in general overate the positive net scattering greenhouse effect of $\mathrm{CO}_{2}$ clouds by allowing more stellar radiation to be transmitted through the cloud layer and more thermal radiation back-scattered to the planetary surface. However, the exact climatic impact will depend on the spectral distributions of the radiation incident on the cloud. These effects are therefore discussed for different central stars and atmospheric temperatures in the following

\section{Radiative impact of $\mathrm{CO}_{2}$ clouds}

By using the results of the radiative transfer calculations with normalised boundary conditions presented in the previous section we now determine the net radiative effect of a given $\mathrm{CO}_{2}$ cloud for different incident stellar spectra and atmospheric temperatures.

We let $F_{\mathrm{s}, \lambda}^{\downarrow}$ denote the downward flux from the central star incident at the top of the atmosphere which can be written as

$F_{\mathrm{s}, \lambda}^{\downarrow}=f_{\mathrm{s}, \lambda} F_{\mathrm{s}}^{\downarrow}$

where $f_{\mathrm{s}, \lambda}$ is the normalised spectral distribution and $F_{\mathrm{s}}^{\downarrow}$ is the total (wavelength-integrated) flux. For simplicity we describe $F_{\mathrm{s}, \lambda}^{\downarrow}=F_{\mathrm{s}, \lambda}^{\downarrow}\left(T_{\mathrm{eff}}\right)$ by black-body radiation with a given stellar effective temperature ${ }^{2} T_{\text {eff }}$. Likewise, the upward thermal radiation incident at the cloud base is denoted by $F_{\mathrm{a}, \lambda}^{\uparrow}$, which can also be factorised in analogy to Eq. (6):

$F_{\mathrm{a}, \lambda}^{\uparrow}=f_{\mathrm{a}, \lambda} F_{\mathrm{a}}^{\uparrow}$

where $F_{\mathrm{a}}^{\uparrow}$ is the wavelength-integrated flux and $f_{\mathrm{a}, \lambda}$ the corresponding normalised spectral distribution. The infrared radiation from the lower atmosphere $F_{\mathrm{a}, \lambda}$ is assumed to be black-body radiation of a given temperature $T_{\mathrm{a}}$. This temperature $T_{\mathrm{a}}$ is not necessarily the surface temperature of the planet but could be any temperature below the cloud base where the atmosphere becomes transparent in the IR.

In the cloud-free case the total amount of incident shortwave radiation being absorbed by the planet is determined as usual by $\left(1-a_{\mathrm{p}}\right) F_{\mathrm{s}}^{\downarrow}$, where $a_{\mathrm{p}}$ is the (cloud-free) planetary albedo (see Fig. 4) given by

$a_{\mathrm{p}}\left(T_{\text {eff }}\right)=\int_{0}^{\infty} a_{\mathrm{p}, \lambda} f_{\mathrm{s}, \lambda} \mathrm{d} \lambda$.

\footnotetext{
2 Note, that non-black-body spectra could, of course, be studied straightforwardly.
}

Conservation of energy implies that this absorbed energy is balanced by the emitted (clear sky - cs) thermal flux $F_{\mathrm{a}, \mathrm{cs}}^{\uparrow}$ :

$F_{\mathrm{a}, \mathrm{cs}}^{\uparrow}=\left(1-a_{\mathrm{p}}\right) F_{\mathrm{s}}^{\downarrow}$.

In contrast to a clear sky, the amount of shortwave radiation absorbed by the planet in the cloudy case (c) is given by

$$
\begin{aligned}
\int_{0}^{\infty}\left(1-a_{\mathrm{p}, \lambda}\right) F_{\mathrm{s}, \mathrm{cb}, \lambda}^{\downarrow} \mathrm{d} \lambda & =\int_{0}^{\infty}\left(1-a_{\mathrm{p}, \lambda}\right) \epsilon_{\mathrm{s}, \lambda} F_{\mathrm{s}, \lambda}^{\downarrow} \mathrm{d} \lambda \\
& =F_{\mathrm{s}}^{\downarrow} \int_{0}^{\infty}\left(1-a_{\mathrm{p}, \lambda}\right) \epsilon_{\mathrm{s}, \lambda} f_{\mathrm{s}, \lambda} \mathrm{d} \lambda,
\end{aligned}
$$

using Eq. (4) for $\epsilon_{\mathrm{s}, \lambda}$ and Eq. (6) for $F_{\mathrm{s}, \lambda}^{\downarrow}$.

Thus, for each considered stellar effective temperature we calculate the total percentage $\epsilon_{\mathrm{s}}$ of the incident shortwave radiation being absorbed by the planet according to

$\epsilon_{\mathrm{s}}\left(T_{\mathrm{eff}}\right)=\int_{0}^{\infty}\left(1-a_{\mathrm{p}, \lambda}\right) \epsilon_{\mathrm{s}, \lambda} f_{\mathrm{s}, \lambda} \mathrm{d} \lambda$,

using an adaptive Gauss-Kronrod quadrature method. On the other hand, the amount of thermal radiation scattered back towards the surface by the cloud is given by (see Eqs. (5) and (7))

$$
\int_{0}^{\infty} F_{\mathrm{a}, \mathrm{cb}, \lambda}^{\downarrow} \mathrm{d} \lambda=\int_{0}^{\infty} \epsilon_{\mathrm{a}, \mathrm{r}, \lambda} F_{\mathrm{a}, \mathrm{c}, \lambda}^{\uparrow} \mathrm{d} \lambda=F_{\mathrm{a}, \mathrm{c}}^{\uparrow} \int_{0}^{\infty} \epsilon_{\mathrm{a}, \mathrm{r}, \lambda} f_{\mathrm{a}, \lambda} \mathrm{d} \lambda .
$$

Therefore, the total percentage $\epsilon_{a, r}$ of the upwelling thermal flux $F_{\mathrm{a}, \mathrm{c}}^{\uparrow}$ back-scattered by the cloud is obtained by

$\epsilon_{\mathrm{a}, \mathrm{r}}\left(T_{\mathrm{a}}\right)=\int_{0}^{\infty} \epsilon_{\mathrm{a}, \mathrm{r}, \lambda} f_{\mathrm{a}, \lambda} \mathrm{d} \lambda$

The wavelength region $(14 \mu \mathrm{m}-16 \mu \mathrm{m})$ around the $15 \mu \mathrm{m}$ absorption band of $\mathrm{CO}_{2}$ is excluded in this integration. Both, the $\mathrm{CO}_{2}$ gas molecules and the $\mathrm{CO}_{2}$ cloud can strongly absorb and re-emit thermal radiation in that particular spectral region, which might result in a classical greenhouse effect. Because the cloud's thermal emission, as well as the absorption and thermal emission by $\mathrm{CO}_{2}$ molecules, is not considered in our radiative transfer calculations (see previous section), this wavelength region is omitted from the analysis of the scattering greenhouse effect. Calculations by, say, Forget \& Pierrehumbert (1997), which also included the $\mathrm{CO}_{2}$ gas, however, showed that the contribution of this spectral region to the greenhouse effect of the $\mathrm{CO}_{2}$ cloud seems to be small.

According to the conservation of energy, the upwelling thermal flux in the cloudy case $F_{\mathrm{a}, \mathrm{c}}^{\uparrow}$ is balanced by the absorbed downward shortwave flux $F_{\text {s,cb }}^{\downarrow}$ and back-scattered thermal flux $F_{\mathrm{a}, \mathrm{cb}}^{\downarrow}$ :

$$
\begin{aligned}
F_{\mathrm{a}, \mathrm{c}}^{\uparrow} & =\int_{0}^{\infty}\left(1-a_{\mathrm{p}, \lambda}\right) F_{\mathrm{s}, \mathrm{cb}, \lambda}^{\downarrow} \mathrm{d} \lambda+\int_{0}^{\infty} F_{\mathrm{a}, \mathrm{cb}, \lambda}^{\downarrow} \mathrm{d} \lambda \\
& =\epsilon_{\mathrm{s}} F_{\mathrm{s}}^{\downarrow}+\epsilon_{\mathrm{a}, \mathrm{r}} F_{\mathrm{a}, \mathrm{c}}^{\uparrow}=\left(1-\epsilon_{\mathrm{a}, \mathrm{r}}\right)^{-1} \epsilon_{\mathrm{s}} F_{\mathrm{s}}^{\downarrow} .
\end{aligned}
$$

We therefore define the ratio of the thermal radiation fluxes of the cloudy $\left(F_{\mathrm{a}, \mathrm{c}}\right.$, Eq. $\left.(15)\right)$ and clear-sky case $\left(F_{\mathrm{a}, \mathrm{cs}}\right.$, Eq. $\left.(9)\right)$ :

$\frac{F_{\mathrm{a}, \mathrm{c}}}{F_{\mathrm{a}, \mathrm{cs}}}=\frac{\epsilon_{\mathrm{s}}}{\left(1-\epsilon_{\mathrm{a}, \mathrm{r}}\right)\left(1-a_{\mathrm{p}}\right)} \quad \begin{cases}>1 & \text { net heating effect } \\ =1 & \text { radiatively neutral } \\ <1 & \text { net cooling effect }\end{cases}$

which gives an indication of the net radiative effect of the cloud layer. For a ratio greater than one, the cloud has a net heating 

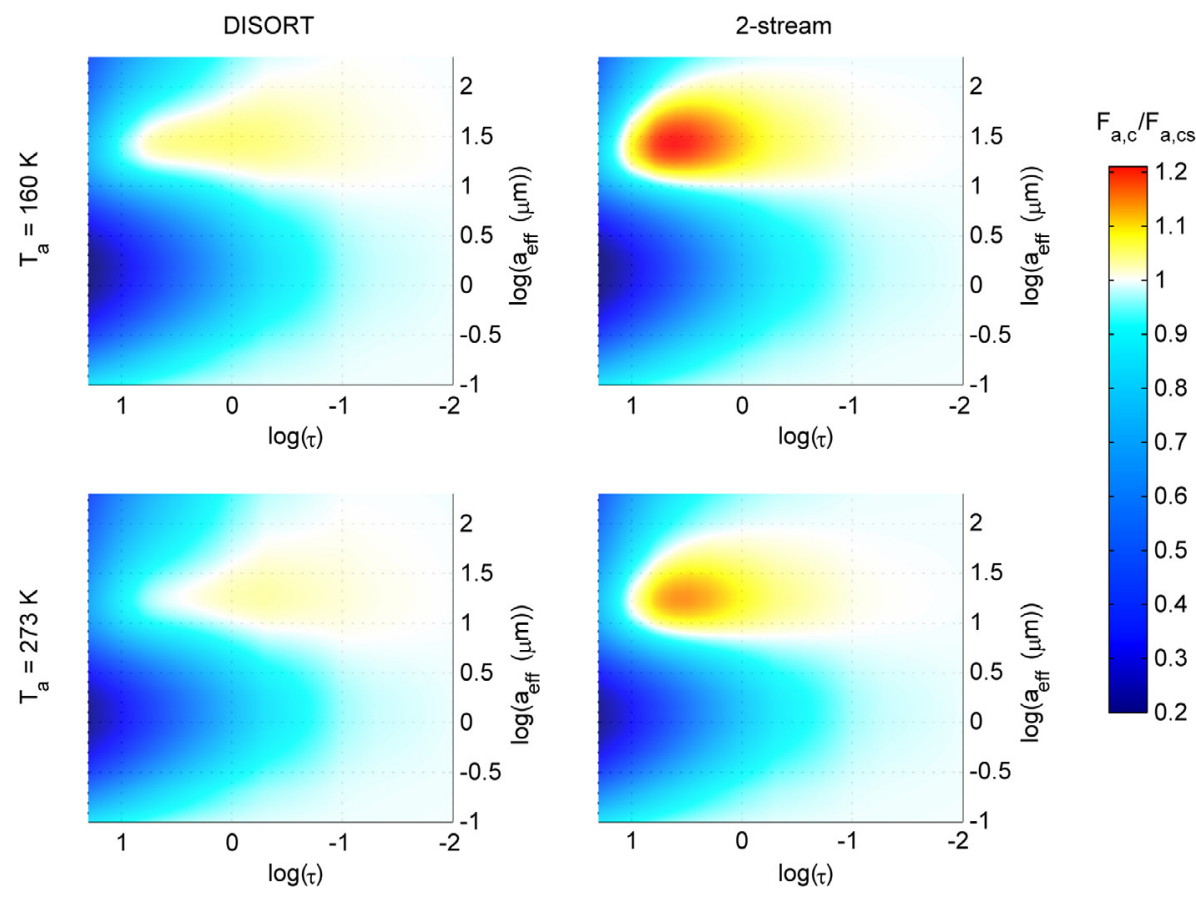

Fig. 6. Ratios $F_{\mathrm{a}, \mathrm{c}} / F_{\mathrm{a}, \mathrm{cs}}$ as a function of optical depth $\tau$ and the effective radius $a_{\text {eff }}$ of the gamma size distribution for an effective temperature of $T_{\text {eff }}=3000 \mathrm{~K}$ and two values of the atmospheric temperatures $T_{\mathrm{a}}: 160 \mathrm{~K}$ (upper $d i$ agrams), $273 \mathrm{~K}$ (lower diagrams). Results are shown for calculations using DISORT (left column) and two-stream methods (right column). The same colour scaling is used for each diagram. (This figure is available in colour in the electronic form.)

effect on the atmosphere below the cloud, while for values lower than one the lower atmosphere is cooled by the cloud layer. A ratio of exactly one represents radiatively neutral cloud particles.

To study the effectiveness of the (net) scattering greenhouse effect, several calculations are performed for a broad range of stellar effective temperatures between $8000 \mathrm{~K}$ and $2000 \mathrm{~K}$. Since cool M-type dwarf stars seem to be particularly important in view of the detectability of (potentially) habitable planets (Rauer et al. 2011), detailed results for a low effective stellar temperature of $3000 \mathrm{~K}$ are presented in the next section.

For the thermal radiation we assume two limiting cases: first we consider the atmosphere below the cloud to be optically thin, such that $T_{\mathrm{a}}$ would correspond to the surface temperature of a habitable planet at the outer boundary of the habitable zone (i.e. $T_{\mathrm{a}}=273 \mathrm{~K}$, freezing point of water). In the second case we consider the atmosphere below the cloud to be opaque such that only thermal radiation directly from below the cloud base reaches the cloud. In this case we adopt a temperature $T_{\mathrm{a}}=160 \mathrm{~K}$ that roughly corresponds to the temperature where $\mathrm{CO}_{2}$ would condense (cf. model calculations of Mischna et al. 2000; or Colaprete \& Toon 2003).

\subsection{Results for a cool M-dwarf star}

In the following we discuss our results for a stellar effective temperature of $3000 \mathrm{~K}$, roughly corresponding to a cool M5 dwarf star. The ratios $F_{\mathrm{a}, \mathrm{c}} / F_{\mathrm{a}, \mathrm{cs}}$ are shown in Fig. 6 as a function of the optical depth $\tau$ and effective particle radius $a_{\mathrm{eff}}$ for both considered atmospheric temperatures. The results of two-stream radiative transfer calculations are also shown for comparison. For a more detailed analysis, different slices through the twodimensional parameter space are shown in Figs. 7 and 8 for several different particle sizes and optical depths.

The results depicted in Figs. 6-8 suggest that the $\mathrm{CO}_{2}$ particles have a negative or neutral impact over a wide range of the considered parameter space. Only particles with sizes comparable to the wavelength of the thermal radiation contribute to a net greenhouse effect if the optical depth is not too large.

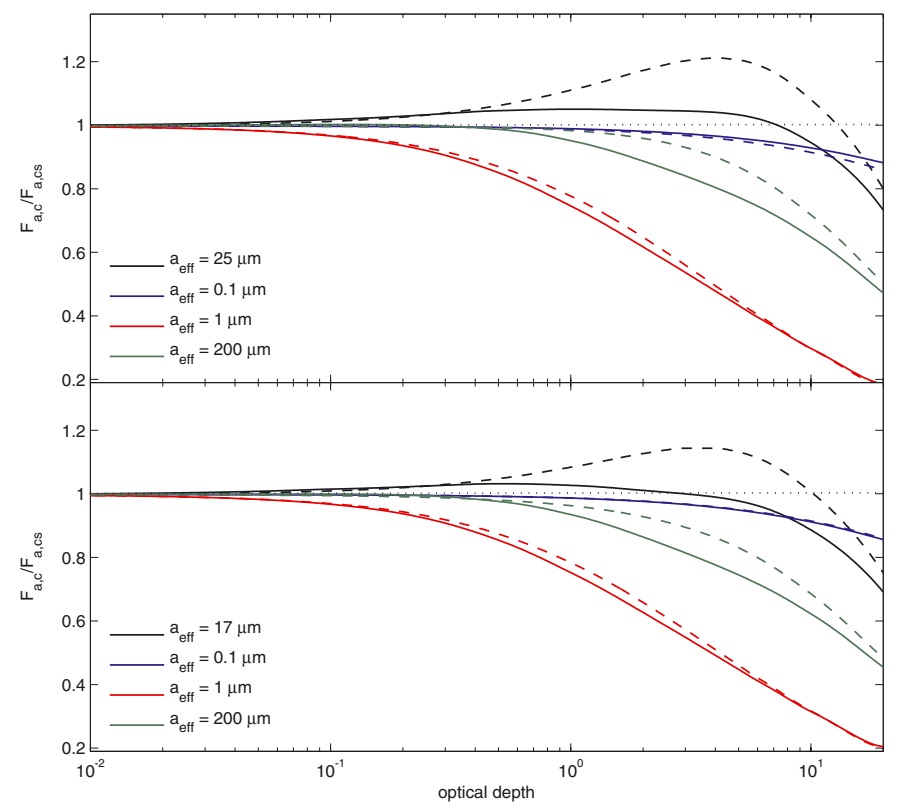

Fig. 7. Ratios $F_{\mathrm{a}, \mathrm{c}} / F_{\mathrm{a}, \mathrm{cs}}$ as a function of optical depth $\tau$ for an effective temperature of $T_{\text {eff }}=3000 \mathrm{~K}$ and the two different atmospheric temperatures: $T_{\mathrm{a}}=160 \mathrm{~K}$ (upper diagram) and $T_{\mathrm{a}}=273 \mathrm{~K}$ (lower diagram). Results are shown for different size distributions in each diagram: $a_{\mathrm{eff}}=25 \mu \mathrm{m}$ (black line, upper diagram), $a_{\mathrm{eff}}=17 \mu \mathrm{m}$ (black line, lower diagram), $a_{\mathrm{eff}}=0.1 \mu \mathrm{m}$ (red line), $a_{\mathrm{eff}}=1.0 \mu \mathrm{m}$ (blue line), and $a_{\text {eff }}=200 \mu \mathrm{m}$ (green line). Calculations using DISORT (twostream methods) are denoted by solid lines (dashed lines).

For higher optical depths, again a cooling effect is found. This kind of behaviour as a function of the optical depth is consistent with the results of Mischna et al. (2000) and Colaprete \& Toon (2003).

Thus, $\mathrm{CO}_{2}$ ice clouds have a certain parameter range of particle radii and optical depths where the heating effect is most efficient. These particle radii are a function of temperature $T_{\mathrm{a}}$ and 


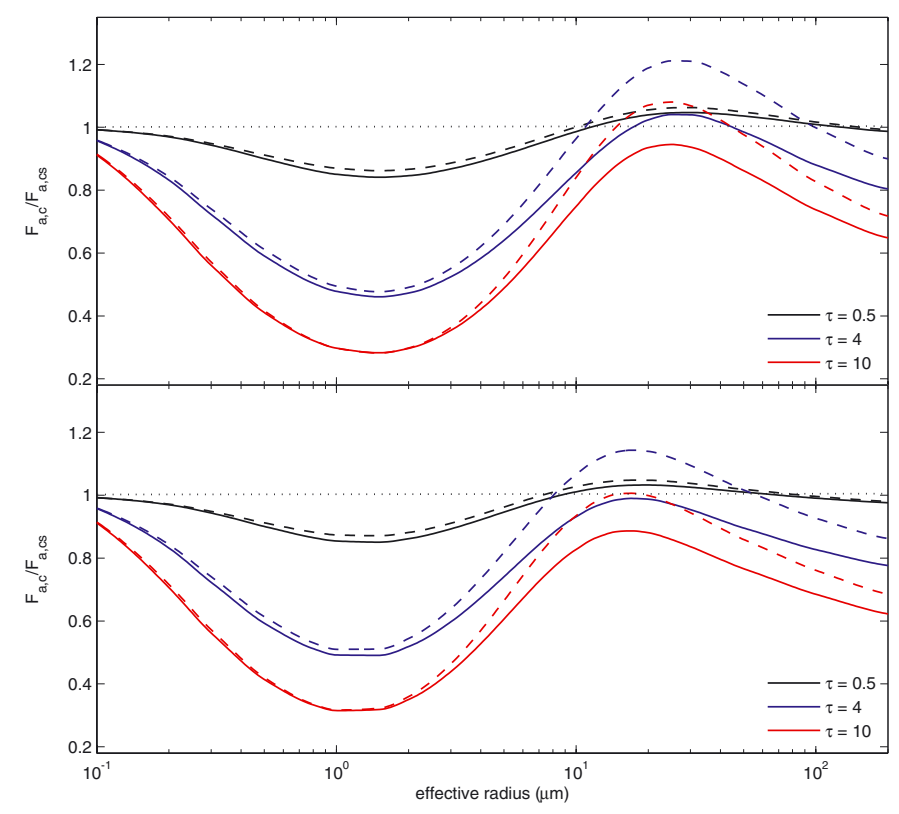

Fig. 8. Ratios $F_{\mathrm{a}, \mathrm{c}} / F_{\mathrm{a}, \mathrm{cs}}$ as a function of effective particle radius $a_{\mathrm{eff}}$ for an effective temperature of $T_{\text {eff }}=3000 \mathrm{~K}$ and the two different atmospheric temperatures: $T_{\mathrm{a}}=160 \mathrm{~K}$ (upper diagram) and $T_{\mathrm{a}}=273 \mathrm{~K}$ (lower diagram). Results are shown for different values of the optical depth $\tau$ in each diagram: $\tau=0.5$ (black line), $\tau=4$ (red line), and $\tau=10$ (blue line). Calculations using DISORT (two-stream methods) are denoted by solid lines (dashed lines).

increase with decreasing temperature; i.e., the most efficient particle radius is about $25 \mu \mathrm{m}(17 \mu \mathrm{m})$ for $T_{\mathrm{a}}=160 \mathrm{~K}\left(T_{\mathrm{a}}=273 \mathrm{~K}\right)$. Apart from the shift in the particle size, the efficiency also increases with lower atmospheric temperature, which leads to a stronger scattering greenhouse effect if the cloud layer were, say, located at higher altitudes within the atmosphere (cf. Mischna et al. 2000). However, even the highest ratios are only slightly larger than 1 ; i.e., the resulting quantitative radiative forcing would be small.

For larger or smaller particle sizes a cooling effect is found that is very strong for particles with effective radii near $1 \mu \mathrm{m}$. This is caused by the large optical depth of these particles at short wavelengths where the maximum of the incident stellar radiation is located (see Sect. 3 and Fig. 3). These kinds of particles would lead to massive cooling if present in the atmosphere.

Very small particles $(0.1 \mu \mathrm{m})$ are almost radiatively neutral, independent of the considered optical depth. Even at $\tau=20$ they show only a weak cooling effect. The reason for this behaviour is that these particles make their main contribution to the optical depth at very short wavelengths (cf. Sect. 3). Since the maximum of the incident stellar radiation for the chosen low effective temperature is located near $\lambda=1 \mu \mathrm{m}$ the impact of these small particles is very small. This effect is similar to the known low efficiency of Rayleigh scattering by the gas molecules for cool M-type stars.

According to the comparison of the different radiative transfer methods (Figs. 6-8) the efficiency of warming by $\mathrm{CO}_{2}$-ice clouds differs quite noticeably for different approximations in the numerical treatment that solves the radiative transfer equation. Consistent with previous reports of Forget \& Pierrehumbert (1997) or Mischna et al. (2000), among others, the two-stream methods predict a strong scattering greenhouse effect at medium optical depths near $\tau=4$. In contrast to this, the application of DISORT, however, results in an almost radiatively neutral cloud.
As already mentioned, only at small optical depths near 0.5 is a slight positive effect found that is much less than the two-stream results. Obviously the largest deviations occur for particle sizes that roughly correspond to the wavelength of the thermal radiation because this is the region where Mie scattering is important. The hemispheric mean two-stream method in particular seems to yield quite inaccurate results compared to a more elaborate discrete ordinate radiative transfer. The errors are also clearly a function of the optical depth. For lower optical depths the differences are small, whereas they are greatest in the region of the most efficient greenhouse effect. The deviations decrease again for more increasing optical depths.

\subsection{Other main sequence stars}

Figure 9 shows the ratio $F_{\mathrm{a}, \mathrm{c}} / F_{\mathrm{a}, \mathrm{cs}}$ for three different stellar effective temperatures: $7300 \mathrm{~K}$ (comparable to an F2V star), $5800 \mathrm{~K}$ (G2V star), and $3000 \mathrm{~K}$ (M5V star). All figures use the same colour scheme to make them directly comparable. Results are only shown for $T_{\mathrm{a}}=160 \mathrm{~K}$. As noted in the previous section, the values of $F_{\mathrm{a}, \mathrm{c}} / F_{\mathrm{a}, \mathrm{cs}}$ would be lower for higher atmospheric temperatures. Additionally, Fig. 10 shows the resulting ratios as a function of the effective temperature for several chosen values of the particles effective radii and optical depths.

The results in Fig. 9 indicate that the most efficient particle size for a net scattering greenhouse effect does not vary with the type of the central star. Rather, it is only a function of the lower atmospheric temperatures $T_{\mathrm{a}}$. As discussed for $T_{\mathrm{a}}=160 \mathrm{~K}$, the corresponding effective particle size is about $25 \mu \mathrm{m}$. As shown in Fig. 10, this particle size yields a small net greenhouse effect for cool stars which increases towards higher effective temperatures. This increase is caused by the higher (clear-sky) planetary albedos due to the stronger Rayleigh scattering at shorter wavelengths.

Other particle sizes, however, exhibit larger variations as a function of the central star temperature. For example, lower ratios of $F_{\mathrm{a}, \mathrm{c}} / F_{\mathrm{a}, \mathrm{cs}}$ are obtained for increasing stellar effective temperatures in case of the particles with $a_{\mathrm{eff}}=0.1 \mu \mathrm{m}$. For cooler stars they are almost radiatively neutral, whereas they show a strong cooling effect for higher stellar temperatures. This is directly related to the optical properties (see Fig. 2) and the resulting transmittance from the radiative transfer calculations. As depicted in Fig. 3 a clear decline at shorter wavelength $(\lambda<1 \mu \mathrm{m})$ is present in the spectral transmittance, which makes the albedo effect more efficient at higher effective temperatures of the central star. The greatest negative impact is still obtained for particle size distributions with effective radii close to $1 \mu \mathrm{m}$. This result is almost independent of the stellar effective temperatures.

As already discussed for the cool M-type star, the two-stream methods again strongly overestimate the positive effect of the $\mathrm{CO}_{2}$ cloud. The ratios $F_{\mathrm{a}, \mathrm{c}} / F_{\mathrm{a}, \mathrm{cs}}$ obtained by DISORT are in every case less than those found by the two-stream methods. Especially at medium optical depths of $\tau=4$, large deviations from the more accurate radiative transfer calculations are found. Here, the two-stream methods yield a large scattering greenhouse effect, which also increases at higher stellar effective temperatures. For cooler stars, a less efficient greenhouse effect is obtained (see also Wordsworth et al. 2011). At smaller optical depths, the differences between the two different radiative transfer methods are again smaller. These results indicate that the scattering greenhouse effect was most likely overrated in all previous model studies of the climatic impact of $\mathrm{CO}_{2}$ clouds. 

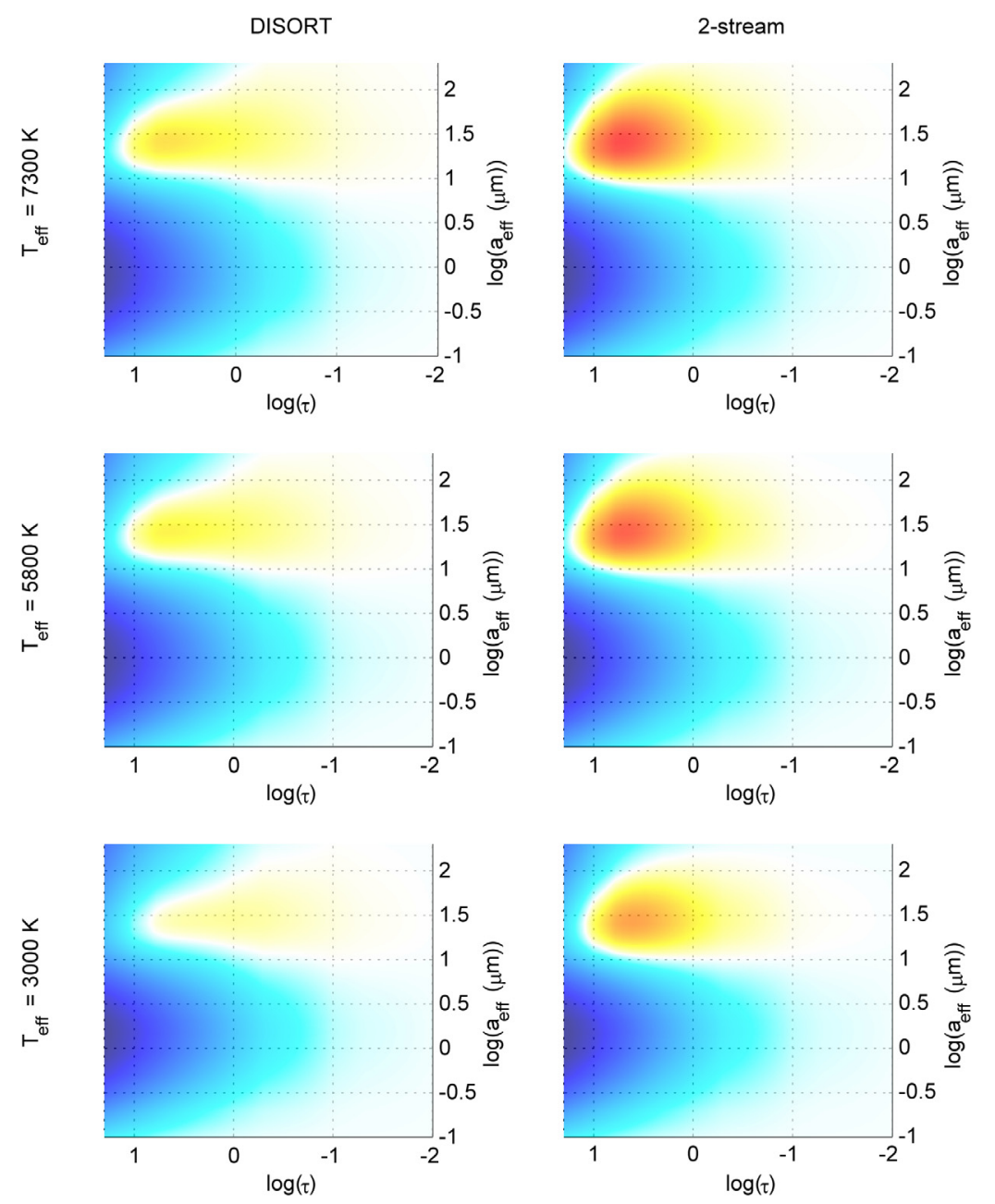

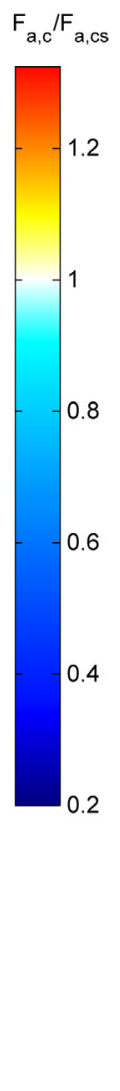

Fig. 9. Ratios $F_{\mathrm{a}, \mathrm{c}} / F_{\mathrm{a}, \mathrm{cs}}$ as a function of optical depth $\tau$ and the effective radius $a_{\text {eff }}$ of the gamma size distribution for three different effective temperatures of the central star: $7300 \mathrm{~K}$ (upper diagrams), $5800 \mathrm{~K}$ (middle diagrams), and $3000 \mathrm{~K}$ (lower diagrams). Results are shown for calculations using DISORT (left column) and two-stream methods (right column). The same colour scheme is used in each diagram. (This figure is available in colour in the electronic form.)

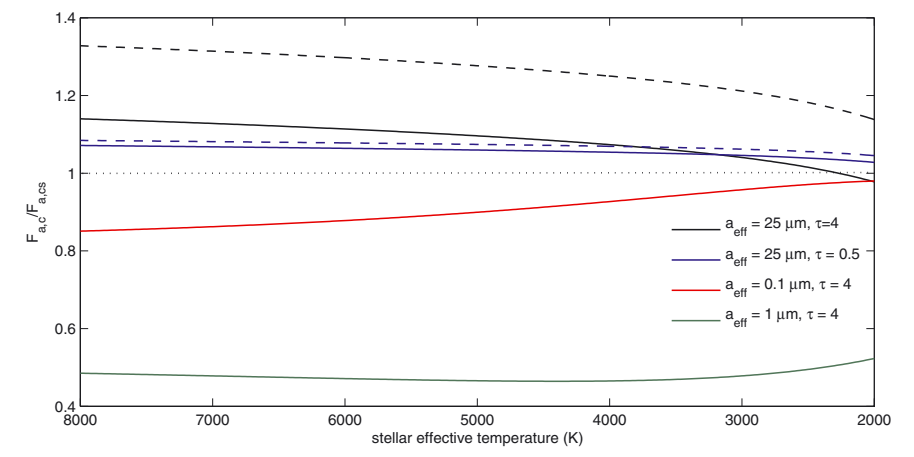

Fig. 10. Ratios $F_{\mathrm{a}, \mathrm{c}} / F_{\mathrm{a}, \mathrm{cs}}$ as a function of the stellar effective temperature. Results are shown for different values of the optical depth $\tau$ and effective radii of the gamma size distributions. Black line: $a_{\mathrm{eff}}=$ $25 \mu \mathrm{m}, \tau=4$, blue line: $a_{\mathrm{eff}}=25 \mu \mathrm{m}, \tau=0.5$, red line: $a_{\mathrm{eff}}=$ $0.1 \mu \mathrm{m}, \tau=4$, green line: $a_{\mathrm{eff}}=1 \mu \mathrm{m}, \tau=4$. Calculations using DISORT are denoted by solid lines, two-stream methods are marked by dashed lines.

\section{Summary}

In this study we investigated the radiative effects of $\mathrm{CO}_{2}$ ice clouds. The particle size distribution were assumed to be gamma distributions with different effective radii. Mie theory was used to calculate the optical properties (optical depths, single scattering albedo, and asymmetry parameter) of $\mathrm{CO}_{2}$ ice particles with effective radii from $0.1 \mu \mathrm{m}$ to $200 \mu \mathrm{m}$. Radiative transfer calculations using different discrete ordinate methods were performed to obtain the spectral reflectance and transmittance of a single layer of $\mathrm{CO}_{2}$ ice particles. In particular, a high-order discrete ordinate solver (DISORT) was employed. Additionally, we also applied simpler two-stream radiative transfer methods for comparison with previous studies. We then studied the net radiative effect of the $\mathrm{CO}_{2}$ ice cloud for different spectral distributions of the incident stellar and the atmospheric thermal radiation.

A net scattering greenhouse effect by $\mathrm{CO}_{2}$ clouds was only obtained for very specific cloud properties. Only particles with sizes comparable to the wavelength of the thermal radiation incident on the cloud base yielded a net greenhouse effect. For larger or smaller particles, a net cooling effect was found. In particular, particles with $a_{\mathrm{eff}} \sim 1 \mu \mathrm{m}$ lead to strong cooling. Very small particles $\left(a_{\mathrm{eff}}=0.1 \mu \mathrm{m}\right)$ were found to be radiatively neutral for very cool stars even at high optical depths. In general, the net heating effect of the $\mathrm{CO}_{2}$ cloud increases with increasing stellar effective temperatures. For cooler stars, however, even the most efficient particle sizes yield only a radiatively neutral cloud.

As suggested by Eq. (15), the planetary surface albedo and the albedo due to molecular Rayleigh scattering can have a strong impact on the net climatic effect of a $\mathrm{CO}_{2}$ cloud. While planets with low surface albedos (e.g. ocean planets) would result in a weaker net heating effect, planets with, e.g., a Martian-like surface albedo can show an increased scattering greenhouse effect. Increasing the amount of $\mathrm{CO}_{2}$ gas, on the other hand, would yield a stronger Rayleigh scattering but also more NIR absorption of the stellar radiation. Thus, for 
central stars with higher effective temperatures one can expect an increased net heating effect by the cloud, while for cooler M-type stars, the $\mathrm{CO}_{2}$ cloud would remain more or less radiatively neutral.

We also compared these results with those of two-stream radiative transfer calculations. Here, a strong greenhouse effect was found at optical depth larger than one and smaller than ten for particle sizes comparable to the wavelength of the thermal radiation, which agrees with, e.g., Forget \& Pierrehumbert (1997) or Mischna et al. (2000). Overall, the two-stream methods yielded large deviations from the calculations using a higher order discrete ordinate method in the important parameter range where a net greenhouse effect was obtained in previous model studies.

Therefore, all these previous studies of the effects of $\mathrm{CO}_{2}$ ice clouds on the outer boundary of the habitable zone that were restricted to two-stream approximations overestimated the positive scattering greenhouse effect. It is evident that more accurate radiative transfer methods are necessary to accurately describe the radiative effects of $\mathrm{CO}_{2}$ clouds. Our study suggests that the scattering greenhouse effect still occurs. However, it is much weaker than previously expected and only efficient for a narrow range of parameters (optical depths, particle sizes). Therefore, we suggest using higher order radiative transfer schemes in future atmospheric model calculations, which include $\mathrm{CO}_{2}$ ice clouds.

In combination with the reduced classical greenhouse effect by $\mathrm{CO}_{2}$ gas molecules due to a revised description of the collisional induced absorption reported by Wordsworth et al. (2010a), this indicates that the outer boundary of the habitable zone should be located closer to the central star than previously considered (e.g. Selsis et al. 2007). A quantitative analysis of the scattering greenhouse effect of $\mathrm{CO}_{2}$ clouds and their impact on the position of the outer boundary of the habitable zone would, however, require an atmospheric model for more detailed calculations.

We used a zenith angle of 60 degree in this study, which corresponds to the global average zenith angle for a onedimensional model. By using smaller zenith angles (e.g. locally in a three-dimensional atmospheric model), one could still obtain higher ratios of $F_{\mathrm{a}, \mathrm{c}} / F_{\mathrm{a}, \mathrm{cs}}$, resulting in a greater scattering greenhouse effect.

Acknowledgements. The authors thank Gary Hansen for providing his refractive index of $\mathrm{CO}_{2}$ ice and Tom Rother, as well as Philip von Paris and Mareike Godolt, for the fruitful discussions. Additionally, we thank the referee Francois Forget for his suggestions for improving the manuscript. This work has been partly supported by the research alliance Planetary Evolution and Life of the Helmholtz Association (HGF).

\section{References}

Baratta, G. A., \& Palumbo, M. E. 1998, J. Opt. Soc. Am. A, 15, 3076

Behnken, H. E. 1912, Phys. Rev. (Series I), 35, 66

Bohren, C. F., \& Huffman, D. R. 1998, Absorption and Scattering of Light by Small Particles (Weinheim: Wiley-VH)

Caldeira, K., \& Kasting, J. F. 1992, Nature, 359, 226

Chandrasekhar, S. 1960, Radiative transfer (Dover Publications Inc.)

Colaprete, A., \& Toon, O. B. 2003, J. Geophys. Res. Planets, 108, 5025

Colaprete, A., Haberle, R. M., \& Toon, O. B. 2003, J. Geophys. Res. Planets, 108,5081

Ehrenfreund, P., Boogert, A. C. A., Gerakines, P. A., et al. 1996, A\&A, 315, L341

Forget, F., \& Pierrehumbert, R. T. 1997, Science, 278, 1273

Forget, F., \& Wordsworth, R. D. 2010, in Pathways Towards Habitable Planets, eds. V. Coudé Du Foresto, D. M. Gelino, \& I. Ribas, ASP Conf. Ser., 430, 55

Forget, F., Hansen, G. B., \& Pollack, J. B. 1995, J. Geophys. Res., 1002, 21219

Glandorf, D. L., Colaprete, A., Tolbert, M. A., \& Toon, O. B. 2002, Icarus, 160, 66

Hansen, G. B. 1997, J. Geophys. Res., 102, 21569

Hansen, G. B. 2005, J. Geophys. Res. Planets, 110, 11003

Henyey, L. G., \& Greenstein, J. L. 1941, ApJ, 93, 70

Hudgins, D. M., Sandford, S. A., Allamandola, L. J., \& Tielens, A. G. G. M. 1993, ApJS, 86, 713

Johnson, B. R., \& Atreya, S. K. 1996, Icarus, 119, 405

Kasting, J. F. 1988, Icarus, 74, 472

Kasting, J. F., Whitmire, D. P., \& Reynolds, R. T. 1993, Icarus, 101, 108

Kitzmann, D., Patzer, A. B. C., von Paris, P., et al. 2010, A\&A, 511, A66

Kramers, H. A. 1927, Atti Cong. Intern. Fisica, 2, 545

Kronig, R. D. L. 1926, J. Opt. Soc. Am., 12, 547

Määttänen, A., Montmessin, F., Gondet, B., et al. 2010, Icarus, 209, 452

Mie, G. 1908, Annalen der Physik, 330, 377

Mischna, M. A., Kasting, J. F., Pavlov, A., \& Freedman, R. 2000, Icarus, 145, 546

Montmessin, F., Gondet, B., Bibring, J.-P., et al. 2007, J. Geophys. Res. Planets, 112, 11

Pierrehumbert, R. T., \& Erlick, C. 1998, J. Atmos. Sci., 55, 1897

Rauer, H., Gebauer, S., Paris, P. V., et al. 2011, A\&A, 529, A8

Selsis, F., Kasting, J. F., Levrard, B., et al. 2007, A\&A, 476, 1373

Stamnes, K., Tsay, S.-C., Jayaweera, K., \& Wiscombe, W. 1988, Appl. Opt., 27, 2502

Toon, O. B., McKay, C. P., Ackerman, T. P., \& Santhanam, K. 1989, J. Geophys. Res., 94, 16287

Vincendon, M., Pilorget, C., Gondet, B., Murchie, S., \& Bibring, J.-P. 2011, J. Geophys. Res. Planets, 116, 0

Wahl, W. 1913, Roy. Soc. London Proc. Ser. A, 89, 327

Warren, S. G. 1986, Appl. Opt., 25, 2650

Warren, S. G., \& Brandt, R. E. 2008, J. Geophys. Res., 26, 14220

Wergin, W. P., Foster, J. L., Chang, A. T. C., et al. 1997, Microsc. Microanalysis, 3,1235

Wiscombe, W. J. 1979, MIE scattering calculations, advances in technique and fast, vector-shaped computer codes, Tech. Rep. (Boulder: NCAR Library)

Wiscombe, W. J. 1980, Appl. Opt., 19, 1505

Wordsworth, R., Forget, F., \& Eymet, V. 2010a, Icarus, 210, 992

Wordsworth, R. D., Forget, F., Selsis, F., et al. 2010b, A\&A, 522, A22

Wordsworth, R. D., Forget, F., Selsis, F., et al. 2011, ApJ, 733, L48 